\title{
Optical turbulence and spectral condensate in long fibre lasers
}

\author{
Elena G. Turitsyna ${ }^{1}$, Gregory Falkovich ${ }^{2}$, Vladimir K. Mezentsev ${ }^{1}$, and Sergei K. Turitsyn ${ }^{1}$
}

(Dated: May 5, 2009)

\begin{abstract}
We study optical wave turbulence using as a particular example recently created ultra-long fibre laser. We show that the sign of the cavity dispersion has a critical impact on the spectral and temporal properties of generated radiation that are directly relevant to the fibre laser performance. For a normal dispersion, we observe an intermediate state with an extremely narrow spectrum (condensate), which experiences an instability and a sharp transition to a strongly fluctuating regime with a wide spectrum and increased probability of spontaneous generation of large amplitude pulses.
\end{abstract}

PACS numbers: 42.65.Sf, 42.55.Ah, 42.55.Wd, 47.10.+g, 47.27.Gs

Powerful fibre laser presents both an interesting nonlinear physical system and a photonic device with an incredible range of practical applications (in telecommunications, medicine, metrology, spectroscopy, sensing, industrial cutting, welding and others). The Raman fibre laser exploits the effect of simulated Raman scattering to shift the generated spectrum from pumping towards longer wavelengths. Using several frequency conversion cascades based on Stokes lines resonating in nested cavities, the pump power (e.g. easily available around one micron) can be shifted deep into the longer wavelength region. Of a particular interest is an ability to fully cover the spectral region near telecommunication windows of transparency, making Raman fibre lasers very attractive pump sources for a distributed Raman amplification that is one of important enabling technologies in high-speed optical communication [1-5]. Using fibre Bragg gratings (FBGs) as cavity reflectors at the Stokes wavelength, it is possible to achieve lasing in a fibre waveguide with the length $L$ of the order of several kilometers [6]. The quasilossless fibre span with highly reduced power variations during transmission can be implemented using symmetric second-order pumping schemes leading to the concept of ultra-long fibre lasers [7-10], which promises new applications in transmission and secure communication [11]. Raman fibre lasers are also attractive continuous light sources for optical coherence tomography [12], long distance remote sensing [13], as a source for the laser guide star for the mesospheric sodium layer [14] and other applications (see e.g. [15]). Despite existing and emerging practical applications of such lasers, some fundamental physical phenomena underlying their operation and nonlinear mechanisms determining properties of generated radiation are not yet fully understood.

In this Letter we demonstrate the impact of optical wave turbulence on spectra and coherence of radiation generated in fibre lasers. Understanding these key properties is very important for practice, and at the same time, presents a fundamental physical problem. A long cavity of a fibre laser provides for a very large number (up to $10^{8}$ ) of interacting modes even for a relatively narrow excitation spectra. That makes such lasers convenient for studying fundamental problems of nonlin- ear and non-equilibrium physics: modulational instability, optical turbulence, interaction of spectral condensate with turbulence. The precision of measurements available in optical experiments makes a fiber laser an excellent tool for quantitative analysis of dynamical and statistical problems of nonlinear physics.

The standard evolution equation for the longitudinal modes (indexed by $m$ ) of the envelope can be derived from the generalized Schrödinger equations for backward and forward Stokes waves [16-18]:

$$
\frac{\tau_{r t}}{L} \frac{d E_{m}}{d t}=\left(G_{m}+i \beta_{2} \Omega_{m}^{2}\right) E_{m}-i \gamma \sum E_{i} E_{k} E_{i+k-m}^{*} .
$$

Here the round trip time is $\tau_{r t}=2 L n / c, L$ is the cavity length and $c / n$ is the speed of light (we use $n=1.45$ ), $G_{m}=g-\delta_{m} / L$ describes the effective pumping $g(P)$ which is a decreasing function of the total generated power $P \equiv \sum\left|E_{m}\right|^{2}$ and the damping $\delta_{m}$ which is a growing function of $m$, see definitions around (2) below. The group velocity dispersion coefficient is $\beta_{2}\left[\mathrm{ps}^{2} / \mathrm{km}\right]$ and $\Omega_{m} \propto m$.

In treating a very large number of interacting modes sharing between them a finite generated power it seemed a natural first step to assume the interaction weak, the spectrum wide and the phases of different modes random. This is a weak-turbulence approach described in [19] and applied to fibre lasers in [17, 18, 20], its predictions are insensitive to the sign of wave dispersion $\beta_{2}$. In the present work, however, we find that changing the dispersion sign dramatically changes the spectral shape and the statistics of the laser radiation.

When the effective pumping/dissipation $G_{m}$ is a decreasing function of both $m$ and $P$, the equation (1) has an exact solution: one-mode condensate, $E_{m}(t)=$ $\sqrt{P} \delta_{m 0} \exp (-i \gamma P t)$, which corresponds (for a given pump) to the maximal $P$ determined by $G_{0}=0$. Side modes $m,-m$ interact resonantly with the zero mode:

$$
\begin{aligned}
d E_{m} / d t & =\left(G_{m}+i \beta_{2} \Omega_{m}^{2}\right) E_{m}-i \gamma P E_{-m}^{*} \\
d E_{-m}^{*} / d t & =\left(G_{m}+i \beta_{2} \Omega_{m}^{2}\right) E_{-m}^{*}-i \gamma P E_{m} .
\end{aligned}
$$

That system has an exponential solution $E_{m}, E_{-m}^{*} \propto$ $\exp (\nu t)$ with $\nu=G_{m}-\beta_{2} \Omega_{m}^{2}\left(\beta_{2} \Omega_{m}^{2}+\gamma P\right)$. Since $G_{m} \leq 0$ 
in a steady state, then only for an anomalous dispersion $\left(\beta_{2}<0\right)$ one can have $\nu>0$ which corresponds to a so-called modulational instability (MI). MI broadens the distribution up to the root-mean square width $\Omega_{r m s}^{2} \simeq \gamma P / \beta_{2}$. Indeed, solving (1) numerically, we observe for $\beta_{2}<0$ the spectra of such width, which are formed and became steady after few round trip times

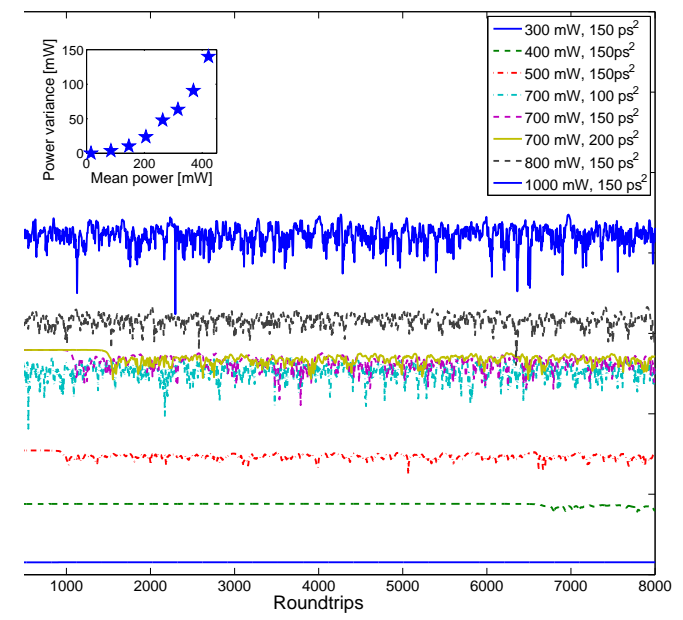

FIG. 1: Generated power versus time for different values of dispersion and pump powers. Inset: power variance versus the mean for $\beta_{2} L=150 \mathrm{ps}^{2}$.

For a normal dispersion, the one-mode condensate is stable with respect to infinitesimal perturbations. In reality, however, the modes of a long cavity are so spectrally dense that one cannot avoid exciting few side modes too. Nonlinear interaction between these few modes can lead to a complicated evolution including dynamical chaos which may result in side modes amplitudes exceeding that of the zero mode at some instant. That may lead to a further spectrum broadening due to so-called fourwave mixing (FWM) described by non-resonant terms in the sum in (1). Indeed, for a range of parameters, we observe that the condensate appears, persists for many round trips and then dissolves into a wider spectrum. That is seen from Figure 1 that shows the evolution of the generated power for $\beta_{2}>0$. A very narrow condensate of few modes is formed initially, it persists for a time depending on the number of modes and the absolute value of dispersion. While during the condensate lifetime the total intensity is constant with high accuracy (Figure 1) it conceals a rich internal dynamics shown in Figure 2 (which corresponds to $700 \mathrm{~mW}$ and $150 \mathrm{ps}^{2}$ ): the interaction between the few modes of the condensate leads to an irregular evolution of their amplitudes which suggest dynamical chaos; this phenomenon and its relation to the condensate destruction will be addressed elsewhere. The condensate destruction is manifested by a sharp transition to a wider spectrum and a lower mean power. That new (statistically steady) state is accompanied by strong fluctuations which seems to be a sign of bi-stability. Inset shows how the fluctuation level depends on the power, it is clear that there is no threshold so that the condensate is likely to be meta-stable at all power levels in that case $\left(\beta_{2} L=150 \mathrm{ps}^{2}\right)$. The condensate life time dramatically increases when the number of modes is small and the dispersion is large; in this case the dispersion between the frequencies of the adjacent modes is large enough to suppress a non-resonant FWM. For sufficiently small number of modes and high $\beta_{2}$ the condensate persisted for as long as we managed to run the simulations, it is unclear whether it is an asymptotic steady state or just a very long-living meta-stable state in such cases.

Spectrum broadening at $\beta_{2}>0$ is due to the competition between FWM and dispersion, so that one can expect that the width is determined by the balance of dispersion and nonlinearity, $\beta_{2} \Omega_{r m s}^{2} \simeq \gamma P$ i.e. comparable to that determined by MI. That would mean that the effective nonlinearity/dispersion ratio $\xi=\gamma P /\left|\beta_{2}\right| \Omega_{r m s}^{2}$ must stay approximately constant and of order unity. We found that indeed $\xi$ practically does not depend on $\left|\beta_{2}\right| L$ (in the interval 50,300 $\mathrm{ps}^{2}$ ) and the pump power (in the interval $\left.2 P_{0}=400,1000 \mathrm{~mW}\right): \xi \simeq 1.7$ for $\beta_{2}<0$ and $\xi \simeq 3.3$ for $\beta_{2}>0$. It is seen that the spectral width is such that the effective dispersion is almost twice larger for $\beta_{2}<0$ when it must balance both nonlinearities, MI and FWM, acting together to widen the spectrum. Since $\xi>1$ then in all these cases most of the modes cannot be treated by the weak turbulence approximation (which is expected to work when $\left.\xi_{m}=\gamma P /\left|\beta_{2}\right| \Omega_{m}^{2} \ll 1\right)$. That conclusion is further supported by the dramatic differences between the cases of normal and anomalous dispersion as discussed above. Figure 3 shows the average spectra of generated radiation after many round trips. While the integral characteristics such as the root-meansquare spectral width are comparable, the spectra for $\beta_{2}<0$ are more smooth and have a characteristic triangular shape with more narrow peaks compared to more concave and irregular spectra for normal dispersion. How an interplay between resonant and non-resonant interactions (MI and FWM) determines so different spectra shapes will be a subject of a future work. The interaction conserves energy so that $\sum G_{m}\left|E_{m}\right|^{2}=0$ in a steady state. Since the effective pumping is a decreasing function of $P$ and $m$, then the broader the steady-state spectrum the larger must be an effective pumping and the smaller is $P$, as indeed seen in Fig. 4 .

Temporal coherence of radiation is characterized by the autocorrelation function $K_{n}(t)=\sum_{\tau}\left\langle E_{n}(\tau-t / 2) E_{n}^{*}(\tau+\right.$ $t / 2)\rangle$. Figure 5 shows $K_{n}(t)$ for negative and positive $\beta_{2}$. The condensate is indeed coherent over many periods while for broad spectra at both signs of $\beta_{2}$ de-correlation happens at the time scale which is below one round trip. 


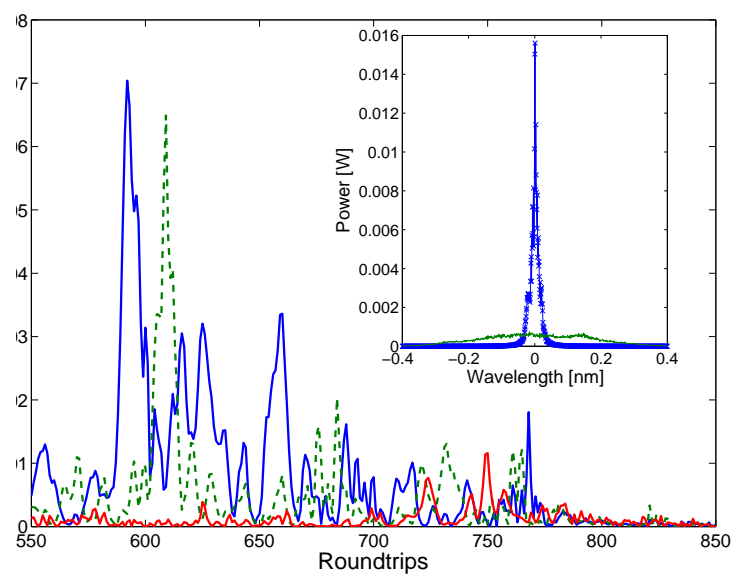

FIG. 2: Time dependence of the modes with $n=0$ (blue), $n=5$ (green) and $n=10$ (red). Inset: spectra before and after condensate destruction: averaged between 550 and 650 roundtrips (blue) and between 850 and 950 roundtrips (green).

\section{$700 \mathrm{~mW}$ total pump power}

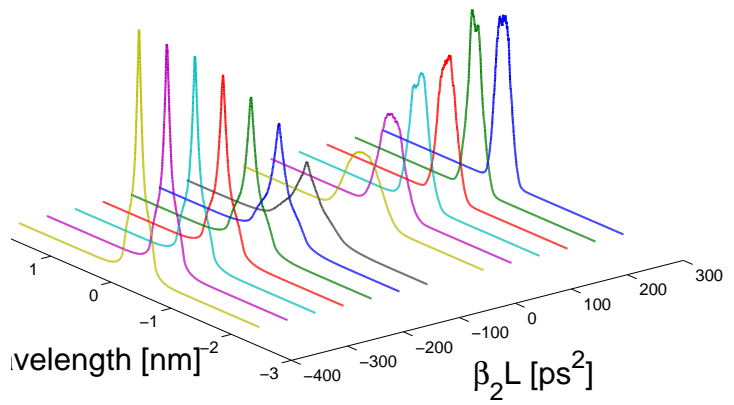

FIG. 3: Spectra of generated radiation for different $\beta_{2} L$.

Fluctuations of the generated power are characterized by histograms in Figure 6 with the condensate distribution being much more narrow than both other histograms. Non-surprisingly, the condensate is the state with less fluctuations, note also that its histogram is very asymmetric with the highest value being most probable which can be naturally interpreted as fluctuations being only "holes" in the condensate (like grey and dark solitons, see e.g. [25]). An abrupt decay of the probability of intensities exceeding certain value can be thought of as an interesting effect of nonlinear self-induced optical limiting, which prevents the laser power from random overshoot and might be of a practical importance in power-sensitive applications in laser systems providing for a stable mode condensate. On the contrary, when the

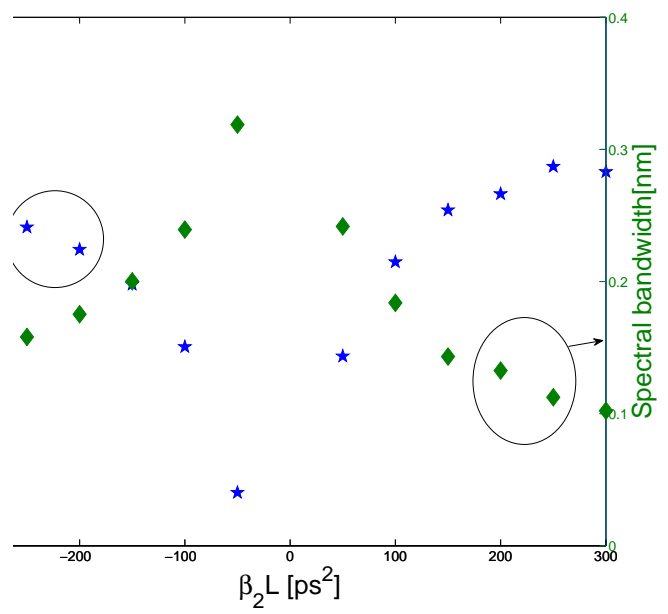

FIG. 4: Spectral bandwidth (diamonds) and generated power (stars) versus $\beta_{2} L$.

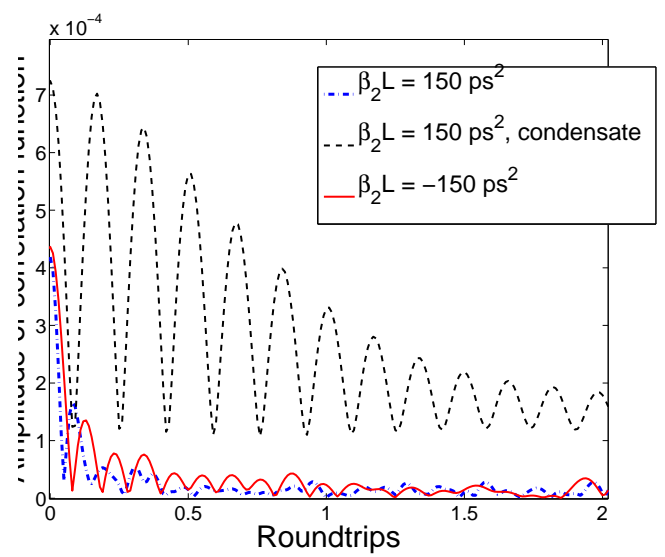

FIG. 5: Amplitude correlation functions for the central mode.

condensate is destroyed, the histograms are much wider and more symmetric with substantial probability of fluctuations exceeding mean value. Somewhat unexpectedly, we see that the modulational instability at $\beta_{2}<0$ broadens the histogram much less than the FWM mixing at $\beta_{2}>0$ (note that while it is reasonable to assume that MI plays a role in creating so-called rogue waves [22$24]$, we see here that the destruction of the condensate produces much higher probabilities of large amplitudes when MI is absent). The important practical observation in Fig. 6 is that positive (normal) cavity dispersion provides for much larger amplitude jitters in the generated continuous wave, compared to the case of negative (anomalous) dispersion.

Here we list the parameters of the numerics. We used the nonlinearity parameter $\gamma=1.45(W k m)^{-1}$ and the effective pumping $g=g_{R} \bar{P}-\alpha$. Here $\alpha=0.25 / 8.66$ $\mathrm{km}^{-1}$ is the distributed fiber loss at $\lambda=1455 \mathrm{~nm}$ (Stokes) and $g_{R} \bar{P}$ is the effective distributed round-trip amplification, which depends on the Raman coefficient 


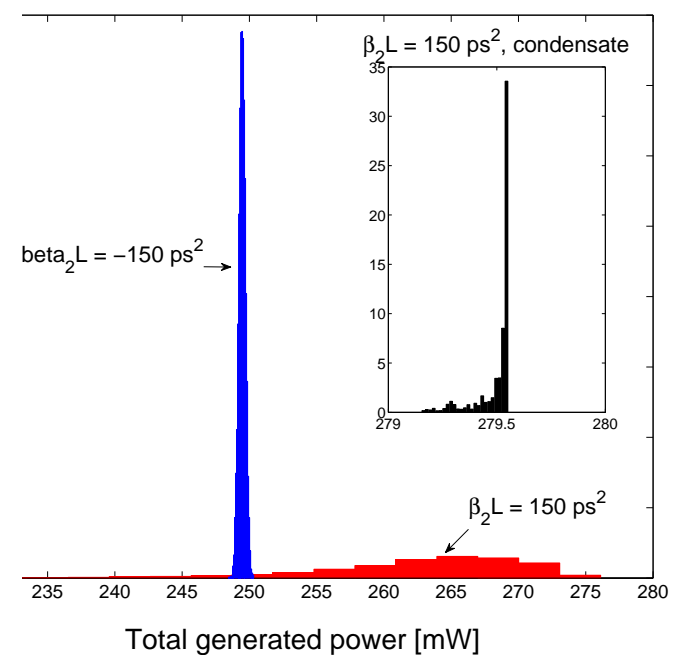

FIG. 6: Histograms of total generated power for normal and anomalous dispersions.

$g_{R} g_{R}=0.51(W k m)^{-1}$ and the pump power $\bar{P}$ averaged over the resonator length (see e.g. [18]):

$$
\bar{P}=2 P_{0} \frac{1-\exp \left[-L\left(\alpha_{P}+\left(\lambda / \lambda_{P}\right) g_{R} P\right)\right]}{L\left(\alpha_{P}+\left(\lambda / \lambda_{P}\right) g_{R} P\right)}
$$

We considered a symmetric pumping scheme described in $[8,10,20], 2 P_{0}$ being the input pump power, $\alpha_{P}=$ $0.31 / 8.66 \mathrm{~km}^{-1}$ is the distributed loss at the pump wavelength $\lambda_{P}=1365 \mathrm{~nm}$. We put $\Omega_{m}=m \Delta$ where $\Delta=\Omega / M$ is a spectral separation between modes, $\Omega$ is the total spectral interval and $M$ is the total number of modes used in numerical modelling. The window of $\Lambda=\lambda^{2} \Omega /(2 \pi c)=8 n m$ significantly exceeded the spectral width in all cases. We used $M=$ $2^{13}$ modes and have verified that changing it up to $2^{16}$, decreasing time steps or increasing the window do not affect the results. $\delta_{m}$ describes the combined effect of all lumped losses and the frequency dependent FBGs losses, we use $\delta_{m}=0.3+\delta_{2}(m \Delta)^{2}=0.3+$ $\delta_{2}\left[\mathrm{~nm}^{-2}\right](\mathrm{m} \Lambda / M)^{2}$ (corresponding to a Gaussian spectral response of the FBGs) with $\delta_{2}\left[\mathrm{~nm}^{-2}\right]=3 \mathrm{~nm}^{-2}$. We used $L=22 \mathrm{~km}$ and the variety of $\beta_{2} L$ values from -300 to $300 \mathrm{ps}^{2}$ (for a single mode fibre with $L=22 \mathrm{~km}$ $\beta_{2} L=-250 \mathrm{ps}^{2}$ ). Input pump power for Figs. 3-6 is $700 \mathrm{~mW}$ for all values of $\beta_{2} L$.

We have here a true multi-mode turbulence with a wide transparency window since for most $m$ within the spectrum $\delta_{m}$ is much less than the effective nonlinearity $\gamma P$, which is comparable with the effective pumping $g$ and dispersion $\beta_{2} \Omega_{r m s}^{2}$ for all values of parameters. We don't expect, however, any turbulence cascades since the interaction is nonlocal in $m$.

To conclude, we have demonstrated that the sign of cavity dispersion has dramatic impact on optical turbu- lence that determines the spectral and temporal properties of generated radiation, directly related to the performance of an ultra-long fibre laser. For a normal dispersion, we observe an intermediate state with an extremely narrow spectrum (condensate) that experiences an instability and a sharp transition to a strongly fluctuating regime. Power histograms show that normal dispersion increases the probability of spontaneous generation of large amplitude pulses - optical rogue waves. For an anomalous dispersion, we have observed triangular spectra and more coherent temporal behaviour of generated radiation.

We acknowledge the support of the Royal Society, EP$\mathrm{SRC}$ and the Israel Science Foundation and the Minerva Einstein Center at WIS.

[1] R. Stolen and E. Ippen, Appl. Phys. Lett. 22, 276 (1972).

[2] L.F. Mollenauer, J.P. Gordon and M.N. Islam, IEEE J. Quant. Elec., vol. QE-22, No. 1, pp. 157-173, Jan. 1986

[3] S.V. Chernikov, A.E. Lewis and J.R. Taylor, OFC'1999, Paper WG6-1, 1999.

[4] C. Headley and G.Agrawal, Raman Amplification in Fibre Optical Communication Systems (Acad. Press 2004).

[5] M. Vasilyev, Proc. Optical Fibre Conf. 2003, OSA. Anaheim,USA, Paper WB1, p. 303 (2003); S. B. Papernyi, V. J. Karpov and W.R.L. Clements, Proc. Optical Fibre Conf. 2002, OSA. Anaheim,USA, Paper FB4 (2002).

[6] S.G. Grubb et al, Proc. of Optical Amplifiers and Their Applications 1995, Proc., Paper SaA4.S7, pp.197-199.

[7] J.D. Ania-Castañón, Opt. Express 12, 4372 (2004).

[8] J.D. Ania-Castañón et al, Phys. Rev. Lett. 96, 023902 (2006).

[9] T.J. Ellingham et al, IEEE Photon. Technol. Lett. 18, 268 (2006).

[10] S.A. Babin et al, Opt. Lett. 32, 1135 (2007).

[11] J. Scheuer and A. Yariv, Phys. Rev. Lett. 97, 140502 (2006).

[12] P.- L. Hsiung et al, Opt. Express 12, 5287 (2005).

[13] Y.-G. Han et al, Opt. Lett. 30, 1114 (2005).

[14] S. Huang et al, Jpn. J. Appl. Phys. 42 L1439 (2003).

[15] N. S. Kim, The Review of Laser Engineering, Supplemental Volume, 1115 (2008).

[16] H.A. Haus and M. Nakazawa, JOSA B 4, 652 (1987).

[17] S.A. Babin et al, Opt. Lett. 31, 3007 (2006).

[18] S.A. Babin et al, JOSA B 24, 1729 (2007).

[19] V.E. Zakharov, V.S. L'vov, G. Falkovich, Kolmogorov spectra of turbulence (Springer-Verlag, 1992).

[20] S.A. Babin et al, Phys. Rev. A, 77(3), 033803 (2008).

[21] P. Janssen, J. Phys. Ocean. 33, 863 (2003)

[22] K. Trulsen and K. B. Dysthe, in Proc. of 21 Symp. Naval Hydrodynamics (Nat. Ac. Press, Washington 1997)

[23] D.R. Solli et al, Nature 450, 1054 (2007)

[24] J.M. Dudley, G. Genty, and B.J. Eggleton, Opt. Express 16, 3644 (2008)

[25] Yu. S. Kivshar, G. P. Agrawal, Optical Solitons: From Fibers to Photonic Crystals (Academic Press, 2003) 